\title{
Layers of fungal complexity
}

DOI:

10.1038/nrmicro1468

\section{URLs} http://www.ncbi.nlm.nih.gov/ entrez/query.

Candida albicans

http://www.ncbi.nlm.nih.gov/ entrez/query.fcgi?db=genome prj\&cmd=Retrieve\&dopt=Over viewElist_uids $=9526$
Entrez Genome Project:

$\mathrm{fcgi}$ ?db=genomeprj

A recent paper in the Journal of Clinical Investigation reports the results of an elegant analysis of the contribution made by the different layers of the Candida albicans cell wall to innate immune stimulation.

The C. albicans cell wall comprises an outer mannoprotein layer containing short chains of $O$-linked mannosyl residues and long, branched chains of $N$-linked mannosyl residues, and an inner layer containing chitin and $\beta$-glucans. Innate immune cells recognize pathogen-associated molecular patterns (PAMPs) using pattern-recognition receptors (PRRs), such as the Toll-like receptors (TLRs) and C-type lectins. Previous work had indicated that components of the mannan layer interact with TLR2, TLR4 and the mannose receptor (MR; a classical C-type lectin) and that $\beta$-glucans are detected by TLR2 and the non-classical C-type lectin dectin 1.

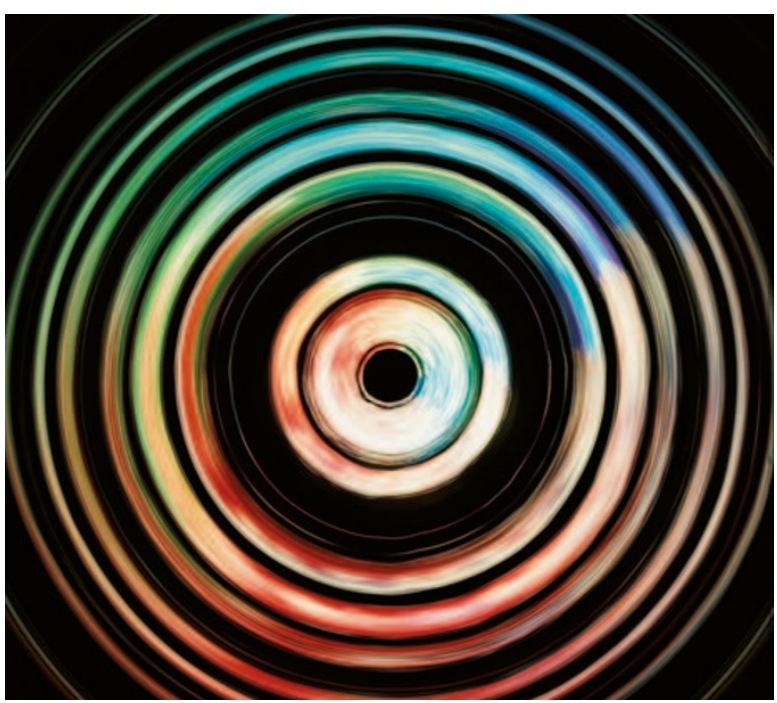

In a systematic genetic approach, Mihai Netea, Neil Gow and colleagues used a combination of C. albicans isogenic mutants, receptor blockade and knockout mice to determine the precise PAMP-PRR interactions involved in C. albicans recognition, and the relative contribution of each interaction. Each of the C. albicans mutants was depleted in a specific cell-wall component: the mnn4 mutant is defective in phosphomannan, the och 1 mutant has a defect in $N$-linked mannosylation, the mnt 1 mnt 2 mutant is defective in O-linked mannosylation, and the pmr1 mutant is defective in both $\mathrm{N}$-linked and $\mathrm{O}$-linked mannosylation. The ability of each mutant strain to stimulate the release of proinflammatory cytokines from human mononuclear cells (MNCs) was analysed. The fact that the pmr 1 mutant showed greatly reduced stimulation compared with wild-type C. albicans initially established the importance of mannosylation. The relative importance of the $\mathrm{N}$-linked and $O$-linked residues was then assessed by comparing the effects of the och1 and mnt1 mnt2 mutants - the absence of $N$-linked mannose residues reduced cytokine production by $70 \%$ whereas the absence of $O$-linked residues reduced it by 15-30\%. The loss of phosphomannan had no effect.

The PAMPs were then matched up with their relevant PRRs by assessing the stimulation of cytokine release in the presence of specific receptor-blocking antibodies. Cytokine release by the och 1 mutant was inhibited by an anti-TLR4 antibody and not an anti-MR antbody and the situation was reversed for the mnt 1 mnt 2 strain. Furthermore, cytokine production in TLR4-deficient mice decreased after stimulation with the och 1 mutant strain but not the mnt 1 mnt 2 strain. Taken together, these results indicate that TLR4 recognizes $O$-linked mannosyl residues and MR recognizes $N$-linked residues.

Finally, the contribution of $\beta$-glucan to immune stimulation was examined by looking at two different scenarios, the stimulation of cytokine release by the och1 mutant in TLR4deficient mice and the stimulation of cytokine release from human MNCs by the mnt 1 mnt 2 mutant in the presence of an anti-MR antibody. In both situations, there should be no immune signal from either $N$-linked or $O$-linked mannosyl residues. Cytokine production was reduced by $\sim 80 \%$ and this residual activity could be removed by the presence of laminarin, a dectin 1 inhibitor.

So, this multilayered approach to a multilayered organism has revealed the presence of three innate immune recognition systems and their differential importance. The results suggest that the $N$-linked mannose-MR interaction is most important, followed by the $O$-linked mannose-TLR4 interaction and then the $\beta$-glucan-dectin 1 interaction, which is consistent with the idea that C. albicans is recognized from the outside in.

Sheilagh Molloy

ORIGINAL RESEARCH PAPER Netea, M. G. et al. Immune sensing of Candida albicans requires cooperative recognition of mannans and glucans by lectin and Toll-like receptors. J. Clin. Invest. 116, 1642-1650 (2006) 